\title{
Increasing light extraction of a substrate emitting OLED using a 2D surface grating.
}

\author{
Peter Vandersteegen*1, Carl Van Buggenhout ${ }^{1}$, Angel Ullan Nieto ${ }^{2}$, \\ Steven Verstuyft ${ }^{1}$, Peter Bienstman ${ }^{1}$, Kristiaan Neyts ${ }^{2}$ and Roel Baets ${ }^{1}$ \\ ${ }^{1}$ Department of Information Technology, UGent-IMEC, St-Pietersnieuwstraat 41,9000 Gent, Belgium \\ ${ }^{2}$ Department of Electronics and Information Systems, UGent, St-Pietersnieuwstraat 41, 9000 Gent, Belgium \\ *e-mail: Peter.Vandersteegen@intec.UGent.be
}

\begin{abstract}
We present simulation and experimental results to achieve increased light extraction of a substrate emitting OLED. A $2 D$ grating located at the glass-air interface experimentally gives a relative improvement of approx. $30 \%$.
\end{abstract}

\section{Introduction}

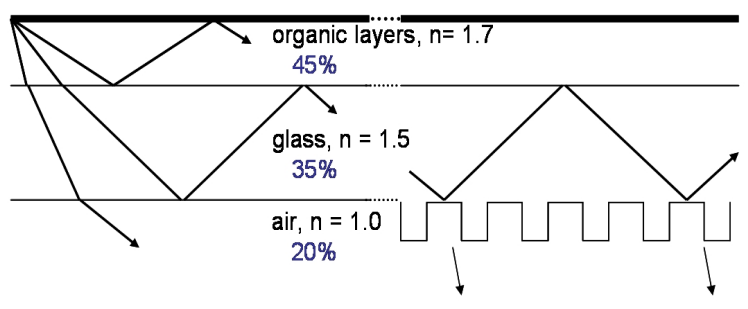

Figure 1: Light is trapped by internal reflection at the interfaces of a planar OLED. The percentages are an estimation of the trapped power in each layer of a structure with planar interfaces.

One promising technology for future general lighting applications is the organic LED (OLED). An OLED typically consists of a glass substrate on which several thin organic films have been deposited between two electrode materials. Light is generated by injecting a current into the organic layers. Extracting this light is limited by total internal reflection (TIR) occurring at several planar interfaces, see fig. 1. All light incident on the organic layer-glass or glass-air interfaces with an angle larger than the critical angle will never be able to escape the structure. Increasing light extraction therefore is crucial to achieve efficient OLEDs.

Placing micro lenses at the glass-air interface results

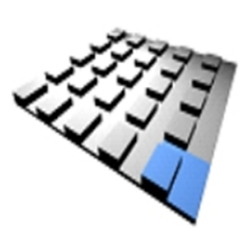

(a)

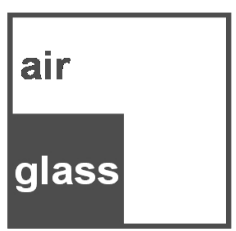

(b)

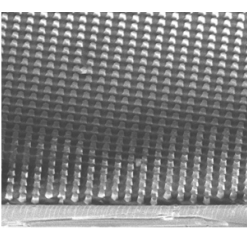

(c)
Figure 2: A 2D grating of pillars. (a) perspective view, (b) one elementary cell and (c) actual fabricated grating.

in a relative improvement of $50 \%,[2]$. This however results in a surface corrugation with a depth of tens of $\mu m$, which might make packaging more difficult. Adjusting the interfaces between the organic layers and the glass substrate, [4], [5] and [6] also can increase light extraction. Placing the corrugation close to the organic layers can negatively influence the light generation.

In this paper we investigate a grating with a depth of only a few $\mu m \mathrm{~s}$ at the glass-air interface. With a simulation tool we numerically optimize an infinite 2D grating placed on the glass substrate of an OLED. Preliminary experimental results also are given.

\section{Theory}

The grating under discussion is shown in figure 2 . The characterizing parameters are the depth, period and fill factor. The last 2 parameters can vary independently in the horizontal and vertical direction of figure 2(b). The simulated OLED layer stack on this glass $(\mathrm{n}=1.528)$ substrate consists of ITO $(\mathrm{n}=1.622$, $100 \mathrm{~nm}) / \operatorname{SiON}(\mathrm{n}=1.806-0.012 \mathrm{j}, 120 \mathrm{~nm}) / \alpha$-NPD $(\mathrm{n}=1.807,30 \mathrm{~nm}) / \mathrm{AlQ3}(\mathrm{n}=1.655,30 \mathrm{~nm})$ and an Al-cathode ( $\mathrm{n}=1.031-6.81 \mathrm{j}, 150 \mathrm{~nm})$.

Our simulation method calculates the light extrac- 
tion for a given wavelength in 2 steps. During the first step the radiant intensity of the organic layer stack is calculated by means of a plane wave expansion of a randomly oriented dipole located between $\alpha$-NPD and AlQ3. This gives us an angular distribution of the radiant flux emitted in glass. The second step convolves the radiant intensity with the angular dependent grating transmission. The grating transmission is defined as the transmission of the power flux for any plane wave incident on the grating, incident at a given angle. This transmission takes into account the multiple reflections, as seen in figure 1 and all power carrying diffraction orders. Contrary to the organic stack, light in the substrate is assumed to behave spatially incoherent. The reflectance and transmittance of the diffracted orders at the grating are calculated with Rigorous Coupled Wave Analysis.

An optimization has been performed for each parameter, while keeping the other parameters constant. This local optimization already gives a good indication for the global optimum, because each grating parameter influences the efficiency largely independently. Best performance is achieved for symmetrical structures, i.e. period and fill factor are equal in both direction of figure 2(b). There is also no wavelength dependency for the optimal fill factor and depth. (optimal: depth $=0.5 \mu m$, fill factor $=70 \%$, period $=$ $1.4-2.0 \mu \mathrm{m})$ The absolute extraction efficiency is also considerably influenced by light absorption of the organic layer stack. If we simulate a organic layer stack which absorbs all incident light, we only see a negligible increase of extraction efficiency compared to a planar structure. This indicates the importance of multiple reflections as sketched in figure 1 .

\section{Experiment}

Fabrication has been done with reactive ion etching of a $\mathrm{SiOx}$ deposited layer on top of a basic glass substrate. The grating pattern of a few tens of $\mathrm{mm}^{2}$ was defined in positive resist with interference lithography.

For our initial experiments, these gratings were fabricated on a different glass substrate than the one on which the OLED stack of $9 \mathrm{~mm}^{2}$ was deposited. Both glass plates were pressed together, using an optical contact fluid to minimize reflections. The total spectral output power was measured with an integrating sphere.

Figure 3 shows the relative improvement in function of the depth both theoretically and experimentally. Our maximum measured relative improvement is $30 \%$ with almost optimal grating parameters. The deviation between simulation and fabrication could be

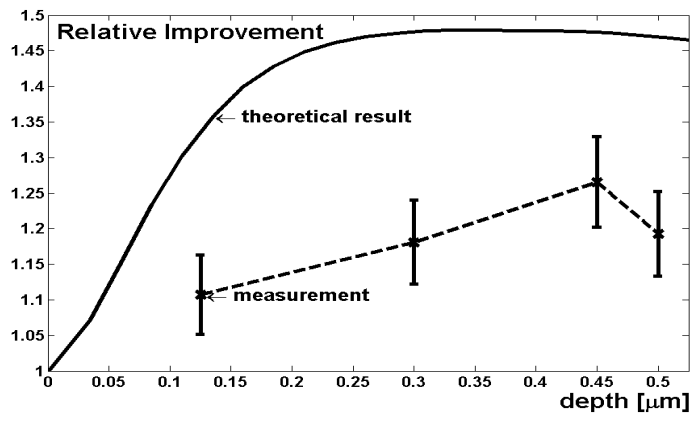

Figure 3: Relative improvement in function of the depth for $\lambda=500 \mathrm{~nm}$, depth $=500 \mathrm{~nm}$, fill factor $50 \%$ and period $=2.0 \mu \mathrm{m}$.

explained by a larger than expected absorption in the organic layer stack.

\section{Summary and Conclusions}

We have demonstrated the feasibility of using a grating at the glass-air layer to increase the light extraction with a surface corrugation depth of approx. $500 \mathrm{~nm}$. A grating numerically achieves a relative improvement of $50 \%$. We have seen a qualitative agreement between simulations and experiments, with a measured $30 \%$ improvement.

\section{Acknowledgements}

The authors thank the European Commission or funding of (part of) this work under contract IST-004607 (OLLA). Peter Bienstman acknowledges the Flemish Fund for Scientific Research (FWOVlaanderen). The authors also wish to thank Horst Greiner of Philips Aachen for the given OLEDs.

\section{References}

[1] N. K. Patel, et al., IEEE J. Select. Topics Quantum Electron., vol.8(2), pp. 346-361, March/April 2002.

[2] S. Möller, et al., J. Appl. Physics, vol.91(5), pp. 3324-3327, Marc 2002.

[3] Toshitaka Nakamura, et al., J. Appl. Physics, vol.96(11), pp. 6016-6022, December 2004.

[4] Jonathan M. Ziebarth, et al., Adv. Func. Mat., vol.14(5), pp. 451-456, May 2004.

[5] Masatoshi Kitamura, et al., Jap. J. Appl. Phys., vol.44(4B), pp. 2844-2848, 2005.

[6] Tetsuo Tsutsui, et al., Adv. Mat., vol.13(15), pp. 1149-1152, August 2001. 


\section{IEEE LEOS Annual Meeting Conference Proceedings}

\section{Copyright and Reprint Permission}

() 2006 IEEE. Personal use of this material is permitted. However, permission to reprint/republish this material for advertising or promotional purposes or for creating new collective works for resale or redistribution to servers or lists, or to reuse any copyrighted component of this work in other works must be obtained from the IEEE.

\section{IEEE Catalog Number: 06CH37736C} ISBN: 0-7803-9556-5

This product was produced for the 2006 IEEE LEOS Annual Meeting Conference by Omnipress. Duplication of this product and its content in print or digital form for the purpose of sharing with others is prohibited without permission from the 2006 IEEE LEOS Annual Meeting Conference.

In no event will Omnipress or its suppliers be liable for any consequential or incidental damages to your hardware or other software resulting from the installation and/or use of this product.

No part of the Omni EZ-Setup ${ }^{\mathrm{TM}}$ software and product "Help" files may be reproduced or used without written permission from Omnipress. (02006 Omnipress - All rights reserved.

For technical issues, contact:

PRODUCED BY

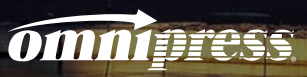

\section{Omnipress Technical Support}

Phone: 608-246-2600

Fax: 608-246-4237

E-mail: digital@omnipress.com

\section{Main Menu}




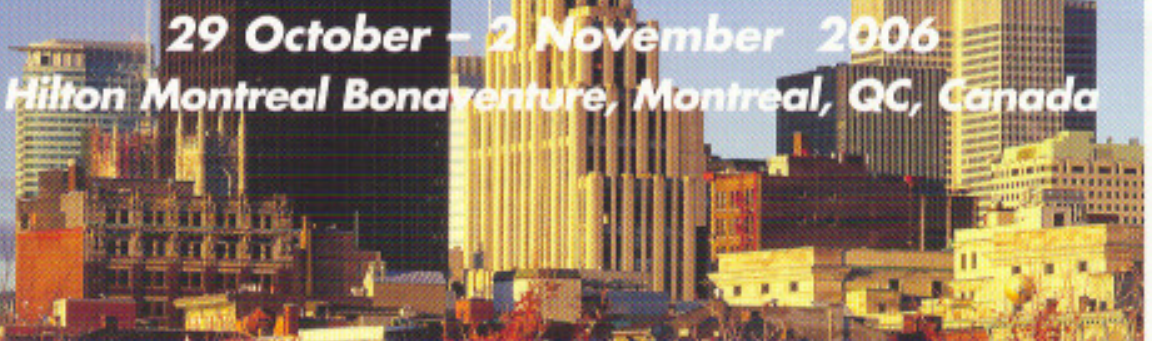

\section{IEEE LEOS Annual Meeting \\ - Conference hoceedings

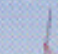

29 October - PVimber

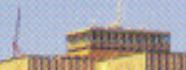

